\title{
Factores asociados con supervivencia a cinco años en niños con astrocitoma cerebral
}

\author{
Ricardo Arturo Sevilla-Castillo y Leticia Aradi Andrade-Sarmiento \\ Instituto Mexicano del Seguro Social, Hospital de Especialidades 25, Servicio de Pediatría Clínica, Monterrey, México
}

\begin{abstract}
Resumen
Introducción: La segunda causa de muerte infantil es el cáncer, del cual las leucemias y los tumores del sistema nervioso central son las presentaciones más frecuentes; de los últimos, los astrocitomas representan $40 \%$. Objetivo: Identificar los factores asociados con la supervivencia a cinco años en niños con astrocitoma cerebral. Método: Estudio de casos y controles anidado en una cohorte retrospectiva en pacientes con diagnóstico de astrocitoma cerebral que recibieron tratamiento de diciembre de 2006 a enero de 2010. Se realizó estadística descriptiva y prueba de chi cuadrado; para evaluar la supervivencia se utilizó el método de Kaplan-Meier. Resultados: Se incluyeron 21 pacientes con diagnóstico de astrocitoma cerebral; la supervivencia fue de $76 \%$ a cinco años, el astrocitoma pilocítico fue el más frecuente (61\%), con una edad media de siete años; predominó el sexo masculino, la edad al fallecimiento osciló entre los ocho y 12 años ( $p=0.022) ; 80 \%$ de los pacientes fallecidos tuvo tumor con localización infratentorial $d(R M=17.3, I C 95 \%=1-622, p=0.01)$. No hubo diferencia en la supervivencia atribuible al tratamiento. Conclusión: La supervivencia a cinco años fue de 76 \%; el factor de riesgo significativo que la limita es la localización infratentorial del tumor.
\end{abstract}

PALABRAS CLAVE: Cáncer infantil. Astrocitoma cerebral. Supervivencia

\begin{abstract}
Introduction: The second cause of children's death is cancer, with leukemia and central nervous system tumors being the most common, among which astrocytomas account for $40 \%$. Objective: To find out the factors associated with 5-year survival in children with cerebral astrocytoma. Method: Case-control study nested in a retrospective cohort of patients diagnosed with cerebral astrocytoma who received treatment from December 2006 to January 2010. Descriptive statistics and the chi-square test were carried out; the Kaplan-Meier method was used to assess survival. Results: Twenty-one patients diagnosed with cerebral astrocytoma were included; survival was $76 \%$ at 5 years; with pilocytic astrocytoma being the most common (61\%); mean age was 7 years, and male gender was predominant; age at death ranged from 8 to 12 years $(p=0.022)$, with $80 \%$ of deceased patients having infratentorial localization $(O R=17.3 ; 95 \% \mathrm{Cl}=1-622 ; p=0.01)$. There was no treatment-attributable survival difference. Conclusions: Five-year survival was $76 \%$; the limiting risk factor is tumor infratentorial localization.
\end{abstract}

KEY WORDS: Pediatric cancer. Cerebral astrocytoma. Surveillance.

\section{Introducción}

La primera causa de muerte en la edad pediátrica son los accidentes, seguidos del cáncer infantil, que representa 0.5 a $5.7 \%$ del total de las neoplasias en la población en general. ${ }^{1}$ Las leucemias constituyen la neoplasia más frecuente en los niños y los tumores del sistema nervioso central (SNC), la segunda. En México, los tumores cerebrales se reportan con una frecuencia de 10.9 a $12 \%$ y tienen una incidencia de
Correspondencia: Ricardo Arturo Sevilla-Castillo

E-mail: ricardo.sevilla @imss.gob.mx
Fecha de recepción: 15-12-2016

Fecha de aceptación: 29-08-2017

DOI://dx.doi.org/10.24875/GMM.17003116
Gac Med Mex. 2018;154:283-286

Disponible en PubMed www.gacetamedicademexico.com 
3.3 a 3.5 casos por cada 100000 niños en centros médicos de tercer nivel y son más frecuente en menores de 10 años, con un pico entre los cuatro y seis años. ${ }^{1-3}$ Los astrocitomas conforman el grupo de tumores originados de la glía; según la Asociación Americana de Cáncer representan $40 \%$ de las neoplasias del $\mathrm{SNC}^{4,5}$ y constituyen el tipo más frecuente. Para evaluar el éxito del tratamiento es necesario determinar la supervivencia; se ha documentado que los pacientes con astrocitomas tienen una supervivencia de $85 \%$ a cinco años.

En el tratamiento del cáncer infantil se evalúan los riesgos y beneficios en cada paciente, a pesar de ello $20 \%$ de los niños fallece y los sobrevivientes tienen efectos negativos en su calidad de vida. ${ }^{6}$ La resección quirúrgica es curativa si se logra de manera total, con una supervivencia de hasta 25 años, a diferencia de lo que sucede con los pacientes en quienes no se logra, ${ }^{7-9}$ en lo cual influye la localización anatómica de la lesión. Se ha comprobado que los pacientes con tumores supratentoriales tienen mejor supervivencia cuando a la cirugía se agrega quimioterapia y radioterapia, esta última con cierta controversia sobre el momento ideal para iniciarla y la dosis recomendada; a pesar de lo anterior se ha comprobado que el inicio temprano de la radioterapia evita la progresión de la tumoración, con lo que se alcanza una mayor supervivencia..$^{10,11}$

Los astrocitomas se dividen en cuatro grados dependiendo del tipo histológico: el pilocítico el más frecuente y el de mejor pronóstico, los tipos más agresivos son el anaplásico y el glioblastoma.

En la actualidad, los tumores cerebrales se diagnostican más pronto gracias a los estudios de imagen con los que se cuenta, sin embargo, en los últimos años se ha documentado aumento en la mortalidad por tumores del SNC, en especial en los seis primeros años del diagnóstico. ${ }^{12,13}$

Con el avance de los estudios diagnósticos y las diferentes modalidades terapéuticas en los tumores cerebrales, es necesario conocer el tiempo esperable de supervivencia y los factores que influyen, particularmente en los astrocitomas cerebrales en niños. El objetivo de nuestro estudio fue evaluar los factores asociados con la supervivencia a cinco años en este grupo de pacientes.

\section{Método}

Estudio observacional, longitudinal, retrospectivo y analítico en el cual se incluyeron todos los pacientes con diagnóstico de astrocitoma del SNC en un periodo

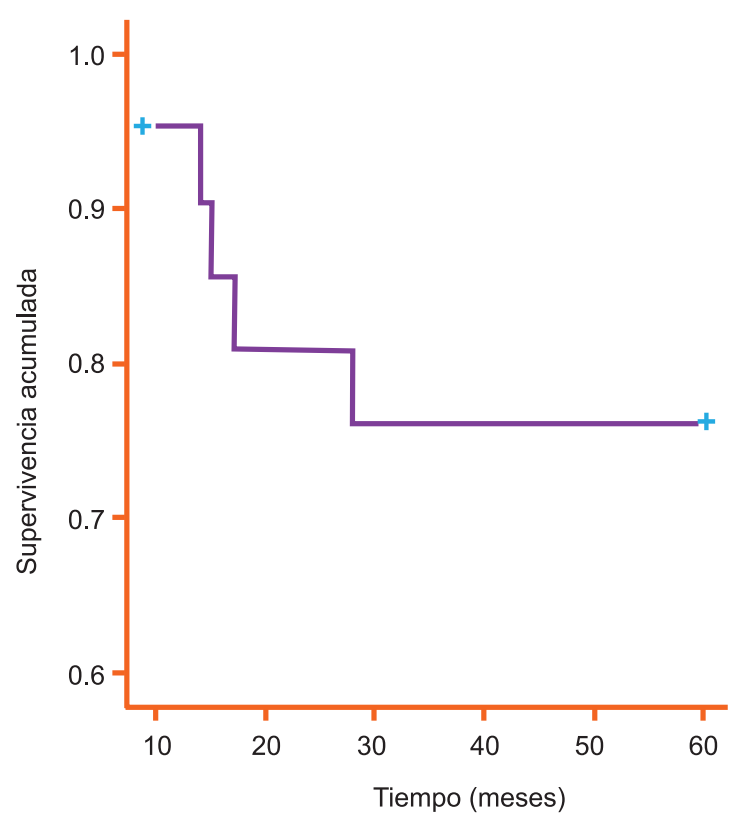

Figura 1. Supervivencia a cinco años de 21 pacientes pediátricos con diagnóstico de astrocitoma.

de cinco años, de enero de 2006 a diciembre a 2010, en el Hospital de Especialidades 25, Monterrey, Nuevo León. Del expediente clínico se obtuvo la siguiente información: sexo, edad al diagnóstico, región anatómica de la localización del tumor, estudio histopatológico, tratamiento administrado, tiempo transcurrido desde el diagnóstico, quimioterapia y el número de sesiones, tratamiento quirúrgico y si se administró o no radioterapia, así como los meses que transcurrieron hasta el fallecimiento. El analizó se llevó a cabo mediante el programa estadístico SPSS versión 17.0. Se utilizó la prueba de chi cuadrado y la prueba de probabilidad exacta de Fisher; para evaluar la supervivencia se utilizó el método de Kaplan-Meier y para evaluar la magnitud de la asociación de los diferentes factores de riesgo se midió la razón de momios (RM) con su intervalo de confianza a $95 \%$ (IC $95 \%$ ), considerando un valor significativo de $p<0.05$.

\section{Resultados}

Se incluyeron 21 pacientes con diagnóstico de astrocitoma cerebral entre enero de 2006 y diciembre de 2010, de los cuales 16 estaban vivos a los cinco años de seguimiento y cinco fallecieron, lo que representó una supervivencia de $76 \%$ (Figura 1). Del total de pacientes, 11 (52\%) fueron del sexo masculino; la población tuvo un rango de edad de dos a 12 años, con una media de siete años; los pacientes que 
Tabla 1. Características clínicas y tratamiento de 21 pacientes pediátricos con astrocitoma de acuerdo con la supervivencia a cinco años

\begin{tabular}{|c|c|c|c|c|c|c|c|}
\hline & \multirow{2}{*}{\multicolumn{2}{|c|}{$\begin{array}{c}\text { Total } \\
(n=21)\end{array}$}} & \multirow{2}{*}{\multicolumn{2}{|c|}{$\begin{array}{c}\text { Vivos } \\
(n=16)\end{array}$}} & \multirow{2}{*}{\multicolumn{2}{|c|}{$\begin{array}{c}\text { Muertos } \\
(n=5)\end{array}$}} & \multirow[t]{3}{*}{$p$} \\
\hline & & & & & & & \\
\hline & $\mathrm{n}$ & $\%$ & $\mathrm{n}$ & $\%$ & $\mathrm{n}$ & $\%$ & \\
\hline \multicolumn{8}{|l|}{ Sexo } \\
\hline Masculino & 11 & 5 & 8 & 50 & 3 & 60 & \multirow[t]{2}{*}{0.702} \\
\hline Femenino & 10 & 48 & 8 & 50 & 2 & 40 & \\
\hline \multicolumn{7}{|l|}{ Estudio histopatológico } & \multirow[t]{5}{*}{0.429} \\
\hline Pilocítico & 13 & 61 & 10 & 62.5 & 3 & 60 & \\
\hline Difuso & 4 & 19 & 4 & 25 & 0 & 0 & \\
\hline Anaplásico & 2 & 9.5 & 1 & 6.2 & 1 & 20 & \\
\hline Glioblastoma & 2 & 9.5 & 1 & 6.2 & 1 & 20 & \\
\hline \multicolumn{8}{|l|}{ Región anatómica } \\
\hline \multirow{3}{*}{$\begin{array}{l}\text { Supratentorial } \\
\text { Infratentorial }\end{array}$} & & & & & & & \multirow{3}{*}{0.013} \\
\hline & 14 & 66 & 13 & 81 & 1 & 20 & \\
\hline & 7 & 33 & 3 & 18 & 4 & 80 & \\
\hline Quimioterapia & 17 & 80 & 12 & 75 & 5 & 100 & 0.225 \\
\hline Radioterapia & 7 & 33 & 5 & 31.2 & 2 & 40 & 0.723 \\
\hline \multicolumn{8}{|l|}{ Resección quirúrgica } \\
\hline Parcial & 12 & 57 & 8 & 50 & 4 & 80 & \multirow[t]{2}{*}{0.248} \\
\hline \multirow[t]{2}{*}{ Total } & 8 & 38 & 7 & 43 & 1 & 20 & \\
\hline & Mediana & Mín.-máx. & Mediana & Mín.-máx. & Mediana & Mín.-máx. & \\
\hline Edad al diagnóstico (años) & 7 & $2-12$ & 6 & $2-11$ & 9 & $8-12$ & 0.022 \\
\hline Tiempo en radioterapia (meses) & 24 & $1-48$ & 6 & $1-48$ & 9 & $1-17$ & 0.769 \\
\hline $\begin{array}{l}\text { Tiempo del diagnóstico a la } \\
\text { cirugía (meses) }\end{array}$ & 1 & $0.5-6$ & 1 & $0.5-3$ & 1 & $0.5-6$ & 0.420 \\
\hline Sesiones de quimioterapia & 7 & $1-14$ & 6 & $1-14$ & 7 & $1-12$ & 0.980 \\
\hline
\end{tabular}

sobrevivieron tuvieron una edad media de seis años, con un rango de dos a 11 años; los que fallecieron tuvieron una media de nueve años, con rango de edad de ocho a 12 años $(p=0.02)$.

De los 21 pacientes con diagnóstico de astrocitoma cerebral, se encontró que $13 \quad(61 \%)$ correspondieron al diagnóstico de astrocitoma pilocítico, cuatro (19\%) a tipo difuso, dos $(9.5 \%)$ a anaplásico y dos (9.5\%) a glioblastoma. En cuanto al sitio anatómico, 14 (66\%) tuvieron una localización supratentorial y siete (33\%) infratentorial. De los pacientes que sobrevivieron, $13(81 \%)$ tuvieron localización supratentorial y tres (18\%) infratentorial; de los que fallecieron, uno (20\%) tuvo localización supratentorial y cuatro $(80 \%)$ infratentorial $(\mathrm{RM}=17.3$, IC $95 \%=1-622, p=0.01)$.

Diecisiete pacientes $(80 \%)$ recibieron tratamiento con quimioterapia, de los cuales $12(75 \%)$ sobrevivieron y cinco (100\%) fallecieron $(p=0.22) ; 20$ pacientes fueron sometidos a procedimiento quirúrgico, de los cuales $12(57 \%)$ tuvieron resección parcial y ocho (38\%), resección total (Tabla 1).

\section{Discusión}

En este estudio se encontró que de todos los tumores del SNC que se diagnosticaron, el astrocitoma se mantuvo con $30 \%$ y fue el más común en el sexo masculino.

Los astrocitomas se diagnostican en $20 \%$ de los pacientes con edades entre los cero y 14 años, pero la edad más frecuente es de los cuatro a seis años. ${ }^{1,3,14}$ Según Bandopadhayay et al., ${ }^{15}$ un factor de mal pronóstico es la edad al diagnóstico: la mortalidad es mayor en los niños < 2 años. Nosotros encontramos que la edad promedio al diagnóstico fue de siete años y que los pacientes que fallecieron tuvieron $<9$ años.

Según la Organización Mundial de la Salud, histológicamente los astrocitomas se clasifican en cuatro grados, siendo el pilocítico el más frecuente y el de 
mejor pronóstico; ${ }^{16}$ nuestros hallazgos correspondieron con los descritos en la literatura mundial. El astrocitoma pilocítico fue la variedad histológica más frecuente en los pacientes que fallecieron: 13 de los 21 pacientes tuvieron esta variedad histológica (61\%) y de los cinco que fallecieron, tres tenían el diagnóstico histológico de astrocitoma pilocítico y la localización fue infratentorial; respecto a los dos pacientes restantes, uno mostró variedad anaplásica y uno, glioblastoma. En 2015, Samaan et al. ${ }^{13}$ observaron alta mortalidad en los primeros dos años de diagnóstico debido a la agresividad del tumor.

En 2008, Osorio et al. ${ }^{17}$ reportaron una supervivencia de $90 \%$ en los pacientes con resección completa de la lesión; en nuestra población no encontramos diferencias entre las diferentes modalidades terapéuticas (quimioterapia, radioterapia y resección quirúrgica) en cuanto a la supervivencia, sin embargo, se encontró que un factor de buen pronóstico fue la localización anatómica del tumor supratentorial; pudiera ser que el beneficio quirúrgico está relacionado con un procedimiento quirúrgico más accesible en la región supratentorial que en la infratentorial. Bandopadhayay et al..$^{15}$ encontraron que el tipo de resección del astrocitoma (parcial o total) no influía en la supervivencia, situación observada en nuestra población.

Las diferentes modalidades de tratamiento como quimioterapia, radioterapia o resección quirúrgica en nuestro estudio no mostró relevancia para la supervivencia como variables independientes, sin embargo, Bandopadhayay et al..$^{15}$ analizaron las bases de datos de los niños que sobrevivieron con diagnóstico de astrocitoma de bajo grado y observaron que cuando la resección fue parcial y recibieron radioterapia, los resultados fueron pobres; concluyeron que probablemente este resultado se debió a los efectos secundarios de la radioterapia. ${ }^{15}$

Nuestra población mostró una supervivencia de $76 \%$, con una mayor probabilidad de morir en los primeros dos años del diagnóstico; al transcurrir los dos años, la posibilidad de mantenerse vivo se extendió a más de cinco años. En un estudio multicéntrico realizado en 1994, Geyer et al. ${ }^{7}$ compararon los tumores anaplásicos y glioblastomas en los primeros dos años del diagnóstico y observaron una supervivencia de $75 \%$; en su investigación efectuada en 2013,
Bandopadhayay et al..$^{15}$ observaron que los pacientes con astrocitoma de bajo grado lograban una supervivencia de $85 \%$; por su parte, la Asociación Americana de Cáncer atribuye una probabilidad de supervivencia de $85 \%$ en niños con astrocitoma cerebral. ${ }^{18}$ Por lo anterior, concluimos que en nuestra población la supervivencia a cinco años fue ligeramente inferior a la documentada en la literatura mundial.

\section{Bibliografía}

1. Secretaría de Salud. Diagnóstico, tratamiento y prevención de los tumores cerebrales infantiles en el primer y segundo nivel de atención. Guía de práctica clínica. México: Secretaría de Salud/Secretaría de la Defensa Nacional/Secretaría de Marina; 2015.

2. Martínez-González MJ, García-Ribes A, Garaizar-Axpe C. Tumores cerebrales infantiles: diagnóstico y semiología neurológica. España: Asociación Española de Pediatría; 2016.

3. Trujillo, C. Basic Concepts of oncology. Medwave. 2003:9(3):e3274.

4. Chico-Ponce-De-León F, Castro-Sierra E, Perezpeña-Diazconti M, Gordillo-Domínguez LF, Santana-Montero BL, Rocha-Rivero LE, et al. Tumores intracraneanos del niño. Bol Med Hosp Infant Mex. 2006;63: 367-381.

5. Hospital Infantil de México Federico Gómez. Astrocitomas de bajo y alto grado: Guías clínicas. México: Hospital Infantil de México Federico Gómez; 2016. Disponible en: http://himfg.com.mx/descargas/documentos/ planeacion/guiasclinicasHIM/AstrocitomasBAG.pdf

6. Smith MA, Seibel NL, Altekruse SF, Ries LA, Melbert DL, O'Leary M, et al. Outcomes for children and adolescents with cancer: challenges for the twenty-first century. J Clin Oncol. 2010;28(15):2625-2634

7. Geyer JR, Finlay JL, Boyyett JM, Wisoff J, Yates A, Mao L, et al. Survival of infants with malignant astrocytomas. A report from the Childrens Cancer Group. Cancer. 1995;75(4):1045-1050.

8. Balmaceda C, Modak S, Finlay J. Central nervous system germ cell tumors. Semin Oncol. 1998;25:243-250.

9. Berger MS, Deliganis AV, Dobbins J, Keles GE. The effect of extent of resection on recurrence in patients with low grade cerebral hemisphere gliomas. Cancer. 1994;74(6):1784-1791.

10. Karim AB, Matt B, Hatlevoll R, Menten J, Rutten EH, Thomas DG, et al. $A$ randomized trial on dose-response in radiation therapy of low-grade cerebral glioma: European Organization for Research and Treatment of Cancer (EORTC) study 22844. Int $\mathrm{J}$ Radiat Oncol Biol Phys. 1996;36(3):549-556.

11. Pollack IF, Hurtt M, Pang D, Albright AL. Dissemination of low grade intracranial astrocytomas in children. Cancer. 1994;77(11):2869-2878.

12. Leibel SA, Sheline GE, Wara WM, Boldrey EB, Nielsen SL. The role of radiation therapy in the treatment of astrocytomas. Cancer. 1975;35: 1551-1557.

13. Samaan MC, Akhtar-Danesh N. The impact of age and race on longevity in pediatric astrocytic tumors: a population-based study. Pediatr Blood Cancer. 2015:62:1567-1571.

14. Secretaría de Salud. Tratamiento de astrocitoma y meduloblastoma en niños y adolescentes en tercer nivel de atención. Guía de práctica clínica. México: Secretaría de Salud/Secretaría de la Defensa Nacional/Secretaría de Marina; 2015.

15. Bandopadhayay P, Bergthold G, London WB, Goumnerova LC, Morales-La Madrid A, Marcus KJ, et al. Long-term outcome of 4,040 children diagnosed with pediatric low-grade gliomas: an analysis of the Surveillance Epidemiology and End Results (SEER) database. Pediatr Blood Cancer. 2014;61(7):1173-1179.

16. Kleihues $P$, Burger PC, Scheithauer BW. The new WHO classification of brain tumours. Brain Pathol. 1993;3(3):255-268.

17. Osorio F, Novoa F, Dalbosco D, et al. Caso clínico: astrocitoma pilocítico. Rev Ped Elec. 2008;5(3):49-53.

18. National Cancer Institute. Surveillance, Epidemiology, and End Results (SEER) Program. Population estimates used in NCl's SEER ${ }^{\star}$ Stat software. Disponible en: http://seer.cancer.gov/popdata/methods.html 